\title{
Mitochondrial genome diversity and evolution in Branchiopoda (Crustacea)
}

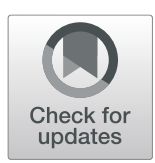

\author{
Andrea Luchetti $^{{ }^{*}}$ D, Giobbe Forni ${ }^{1}$, Alyza M. Skaist ${ }^{2}$, Sarah J. Wheelan ${ }^{2}$ and Barbara Mantovani ${ }^{1}$
}

\begin{abstract}
Background: The crustacean class Branchiopoda includes fairy shrimps, clam shrimps, tadpole shrimps, and water fleas. Branchiopods, which are well known for their great variety of reproductive strategies, date back to the Cambrian and extant taxa can be mainly found in freshwater habitats, also including ephemeral ponds. Mitochondrial genomes of the notostracan taxa Lepidurus apus lubbocki (Italy), L. arcticus (Iceland) and Triops cancriformis (an Italian and a Spanish population) are here characterized for the first time and analyzed together with available branchiopod mitogenomes.

Results: Overall, branchiopod mitogenomes share the basic structure congruent with the ancestral Pancrustacea model. On the other hand, rearrangements involving tRNAs and the control region are observed among analyzed taxa. Remarkably, an unassigned region in the L. apus lubbocki mitogenome showed a chimeric structure, likely resulting from a non-homologous recombination event between the two flanking trnC and trnY genes. Notably, Anostraca and Onychocaudata mitogenomes showed increased GC content compared to both Notostraca and the common ancestor, and a significantly higher substitution rate, which does not correlate with selective pressures, as suggested by $\mathrm{dN} / \mathrm{dS}$ values.

Conclusions: Branchiopod mitogenomes appear rather well-conserved, although gene rearrangements have occurred. For the first time, it is reported a putative non-homologous recombination event involving a mitogenome, which produced a pseudogenic tRNA sequence. In addition, in line with data in the literature, we explain the higher substitution rate of Anostraca and Onychocaudata with the inferred GC substitution bias that occurred during their evolution.
\end{abstract}

Keywords: Branchiopoda, Mitochondrial genomics, Mitochondrial unequal recombination, Notostraca, Nucleotide compositional bias, Nucleotide substitution rate

\section{Background}

The class Branchiopoda is a small but highly diverse group of crustaceans, distributed on all continents and inhabiting both marine and inland waters, including hypersaline lakes and ephemeral or freezing ponds [1-4]. The origin of branchiopods dates back to the Middle Cambrian [5] and the class includes forms retaining remarkably conserved ancestral characters, like the so-called "living fossils" genera Triops and Lepidurus (tadpole shrimps of the order Notostraca).

The class taxonomy has been extensively revised during the last decades and it is presently considered to include four orders: Anostraca (fairy shrimps), Notostraca (tadpole shrimps), Laevicaudata (clam shrimps), and Onychocaudata (Spinicaudata [clam shrimps] + Cyclestherida [clam

\footnotetext{
* Correspondence: andrea.luchetti@unibo.it

${ }^{1}$ Department of Biological, Geological and Environmental Sciences, University of Bologna, via Selmi 3, 40126 Bolgna, Italy

Full list of author information is available at the end of the article
}

shrimps] + Cladocera [water fleas]) [3]. A clear phylogenetic picture of within-Branchiopoda relationships emerged recently with phylogenomic analyses, nicely reconciling with morphological hypotheses. These analyses supported Anostraca as the sister group to Phyllopoda, a clade including all other branchiopods, in which Notostraca are the sister group to Diplostraca (=Laevicaudata + Onychocaudata) $[4,6-8]$.

The relevance of mitochondrial genomics analyses encompasses many fields of biology, ranging from comparative biology studies, to medically related questions and cellular molecular biology [9]. On the other hand, the greatest contribution of mitogenomics has probably been in the fields of molecular phylogeny and evolution $[10,11]$. The animal mitochondrial genome is a relatively simple molecule, with a general structure that is strongly conserved across the animal kingdom. It is circular, approximately 16 , $000 \mathrm{bp}$ long, with 13 protein coding genes (PCGs), two

(c) The Author(s). 2019 Open Access This article is distributed under the terms of the Creative Commons Attribution 4.0 International License (http://creativecommons.org/licenses/by/4.0/), which permits unrestricted use, distribution, and 
rRNA genes, 22 tRNA genes, and a non-coding region of variable length, known as control region [10]. Nonbilaterian animal taxa show remarkable exceptions to this organization [12], with instances of variation in length and gene content. Gene rearrangements are also present in bilaterian taxa (mainly duplications and translocations), with the remarkable instance of the phylum Mollusca [13, 14].

Although generally well-conserved, arthropod mitogenomes also exhibit some variations, ranging from rearrangements of the gene order in some crustaceans $[15,16]$ and insects [17], to duplications of one or a few tRNA genes [18-20] or of the control region [17]. The study of mitogenomic gene arrangement uncovered a higher plasticity of the organization of this molecule than previously thought [13]. Moreover, it also serves as a tool for both improving phylogenetic estimation [11] and understanding mechanisms responsible for mitogenome structural variation [21].

In this article, we report on the sequencing and analysis of four mitogenomes from three taxa of Notostraca. In particular we obtained, for the first time, the whole mitochondrial genomes of two Lepidurus taxa: the Mediterranean form $L$. apus lubbocki and the arctic species $L$. arcticus [22]. Moreover, although the Triops cancriformis mitogenome has previously been sequenced from a sample collected in an invasive population in Japan [23], we here present mitogenomes from two distinct, native populations of $T$. cancriformis with different reproductive modes: a parthenogenetic population (collected in Italy) and a gonochoric one (from Spain) [24]. Finally, newly obtained mitogenomes are also analyzed with all currently available branchiopod mitogenomes in order to draw insights into mitogenome evolution in this class of crustaceans.

\section{Methods}

Total DNA was extracted from individual specimens using the DNA extraction kit (STRATEC), after dissection for gut removal. Whole genome sequencing has been carried out on Illumina HiSeq2000 (Johns Hopkins University Experimental and Computational Genomics Core, SKCCC, USA) on parthenogenetic T. cancriformis sample (paired end, $2 \times 100 \mathrm{bp}$; insert size $=150 \mathrm{bp}$; Luchetti, unpublished data), and on HiSeqX platform (Macrogen Inc., South Korea) on bisexual $T$. cancriformis and Lepidurus taxa (paired end, $2 \times 150 \mathrm{bp}$; insert size $=350 \mathrm{bp}$ ) [25]. Mitochondrial genomes were de novo assembled from a random subset of 1 million read pairs per sample using the SPAdes v. 3.11.1 assembler with default parameters [26]. Coverage was calculated by mapping raw reads with Bowtie2 [27] and analyzing the output with SAMtools [28]. Annotation was de novo performed using the MYTOS pipeline [29] and manually corrected by similarity with the published T. cancriformis mitochondrial genome (GenBank accession number: AB084514). Genes for tRNAs were confirmed by means of ARWEN v. 1.2.3 [30].
A selection of sequenced mitochondrial genomes of branchiopods, and two outgroup insect sequences, were downloaded from GenBank (Table 1). Alignments of PCGs and of rRNA genes were performed with MAFFT v. 7.205 [47], using the automatic detection of parameter set. Alignments of PCGs were carried out considering the amino acid translation to correctly align codons. Alignments for ribosomal RNA genes ( $r r n L$ and $r r n S$ ) were filtered using Gblocks v. $0.91 \mathrm{~b}$ [48], with options for less stringent selection (min. Number of sequences for a conserved position $=22$; $\mathrm{min}$. Number of sequences for a flanking position $=22$; $\max$. Number of contiguous non-conserved positions $=8$; $\min$. Length of a block $=5$; allowed gap positions $=$ with half). All alignments were concatenated, and the best partition scheme and substitution models were estimated using Partition Finder v. 1.1.1 [49]. The best partition schemes and best-fitting substitution models are reported in Additional file 3: Table S1. Maximum-likelihood trees were obtained using RAxML v. 8.2 [50], using the rapid bootstrap option with 500 replicates for nodal support. Bayesian Inferences were obtained with MrBayes v. 3.2.3 [51]. Two tree searches were run for 10,000,000 generations and sampling every 1000 trees. Convergence was assessed by average standard deviation of split frequencies $<0.01$. The BI tree was summarized after a conservative burnin $=25 \%$.

The hypothesis of constancy of substitution rate throughout the tree was tested by comparing the $-\ln L$ of tree with and without strict clock constraint by means of likelihood ratio test, as implemented in MEGA v.7 [52]. A time tree was also calculated by means of BEAST v. 1.8 [53]: two independent chains were run for 60,000,000 generations each and trees sampled every 1000 generations. Mixing and convergence of the two runs was reached when effective sample size of each parameter $>200$. The maximum-cladecredibility tree was determined after discarding the first $10 \%$ of the trees obtained (burnin). Clock model was set to a lognormal uncorrelated relaxed clock, and a birth-death speciation model was assumed. Calibrations were set on crown groups, using a lognormal probability distribution, based on detailed fossils description given in Wolfe et al. [54]: Notostraca were dated using Chenops yixianensis fossil taxon; minimum hard bound =121.8 Mya, maximum soft bound = 521 Mya; Cladocera were dated using Smirnovidaphnia smirnovi fossil taxon; minimum hard bound = 173.1 Mya, maximum soft bound $=521$ Mya; Anostraca were dated using Palaeochirocephalus rasnitsyni fossil taxon; minimum hard bound =125.7 Mya, maximum soft bound = 521 Mya. Branchiopoda were dated using Lepidocaris rhyniensis fossil taxon; minimum hard bound $=407.6$ Mya, maximum soft bound = 521 Mya.

Nucleotide composition was calculated with MEGA v.7. Variation of GC content between clades and ancestor was evaluated on the phylogenetic dataset (PCGs+rRNAs). Estimates of ancestral GC content were obtained by 
Table 1 Samples obtained from Genbank for comparative and phylogenetic analyses

\begin{tabular}{|c|c|c|c|c|c|}
\hline \multicolumn{3}{|l|}{ Taxonomy } & \multirow{2}{*}{$\begin{array}{l}\text { Species } \\
\text { Artemia franciscana }\end{array}$} & \multirow{2}{*}{$\frac{\text { Genbank acc. Nos. }}{\text { X69067 }}$} & \multirow{2}{*}{$\frac{\text { Reference }}{[31]}$} \\
\hline Crustacea & Branchiopoda & Anostraca & & & \\
\hline Crustacea & Branchiopoda & Anostraca & A. tibetiana 1 & JQ975177 & [32] \\
\hline Crustacea & Branchiopoda & Anostraca & A. tibetiana 2 & JQ975178 & [32] \\
\hline Crustacea & Branchiopoda & Anostraca & A. urmiana & JQ975176 & [32] \\
\hline Crustacea & Branchiopoda & Anostraca & Phallocryptus tserensodnomi & KP273592 & [33] \\
\hline Crustacea & Branchiopoda & Anostraca & Streptocephalus sirindhornae & KP273593 & [34] \\
\hline Crustacea & Branchiopoda & Cladocera & Daphnia carinata & KP721459 & [35] \\
\hline Crustacea & Branchiopoda & Cladocera & D. magna & KP296147 & [36] \\
\hline Crustacea & Branchiopoda & Cladocera & D. pulex & AF117817 & [37] \\
\hline Crustacea & Branchiopoda & Cladocera & D. pulex (China) & KT003819 & [38] \\
\hline Crustacea & Branchiopoda & Cladocera & D. galeata & LC177070 & [39] \\
\hline Crustacea & Branchiopoda & Cladocera & Diaphanosoma dubium & MG428405 & [40] \\
\hline Crustacea & Branchiopoda & Spinicaudata & Limnadia lenticularis & MH618637 & [41] \\
\hline Crustacea & Branchiopoda & Notostraca & Triops australiensis & LK391946 & [42] \\
\hline Crustacea & Branchiopoda & Notostraca & T. cancriformis (Japan) & AB084514 & [23] \\
\hline Crustacea & Branchiopoda & Notostraca & T. longicaudatus 1 & AY639934 & [43] \\
\hline Crustacea & Branchiopoda & Notostraca & T. longicaudatus 2 & GU475465 & [44] \\
\hline Crustacea & Branchiopoda & Notostraca & T. longicaudatus "I" & KM516710 & {$[45]$} \\
\hline Crustacea & Branchiopoda & Notostraca & T. longicaudatus "s" & KM516711 & {$[45]$} \\
\hline Crustacea & Branchiopoda & Notostraca & T. newberry & KM516712 & [45] \\
\hline Hexapoda & Insecta & Thysanura & Thermobia domestica & AY639935 & [43] \\
\hline Hexapoda & Insecta & Isoptera & Reticulitermes flaviceps & KX712090 & [46] \\
\hline
\end{tabular}

reconstructing ancestral sequences at Anostraca and Onychocaudata/Notostraca stem node using FastML web server [55] (available at http://fastml.tau.ac.il/, last accessed November 2018). The search was set as follows: PCGs+rRNA alignment were analyzed using the maximum-likelihood tree as guide tree, with GTR + G substitution model and branch lengths optimization. Following the recommendation of Matsumoto et al. [56], we sampled 100 ancestral sequences (Additional files 1 and 2) from the posterior distribution at the two selected stem nodes and obtained average GC values to compare with GC content of extant taxa.

Different substitution rates among clades were tested using the Two-Cluster test, implemented in LINTRE [57]. A tree file formatted for LINTRE was manually built using branch lengths from ML trees and used as input for the Two-Cluster test analysis.

Tests for positive selection were carried out by analyzing the $\mathrm{dN} / \mathrm{dS}$ ratio $(\omega)$ over branches in the ML obtained on PCGs only, using the codeml algorithm implemented in PAML 4.8 [58]. We tested two different models: the oneratio model, assuming a single $\omega$ value for the whole tree (codeml model $=0$ ) and the free-ratios model, assuming a $\omega$ value for each branch (codeml model $=1)$. Finally, a likelihood-ratio test was used to calculate the best fitting model.

\section{Results and discussion}

\section{Mitochondrial genome characterization}

Assembled mitochondrial genomes ranged in length from $15,158 \mathrm{bp}$ (T. cancriformis) to $15,635 \mathrm{bp}$ (L. apus lubbocki) (Table 2; Fig. 1). The overall mitogenome structure is congruent with the ancestral Pancrustacea model [10], showing 13 PCGs, two rRNA genes and 22 tRNA genes (Fig. 1; Additional file 3: Table S2).

The average GC content (Table 2) ranges from $27.8 \%$ (L. apus lubbocki) to 32.5\% (L. arcticus). PCGs are more GC-rich than rRNA and tRNA genes only in L. arcticus; the control region of $L$. apus lubbocki is the most ATrich region among the three taxa.

Nine out of the 13 PCGs (atp6, atp8, cox1, cox2, cox3, cob, nad2, nad3, nad6) are encoded on the $\mathrm{H}$ strand, while four are encoded on the L strand (nad1, nad4, nad4L, nad5). In T. cancriformis, most PCGs have ATG $(n=6)$ or ATA $(n=5)$ start codons; the nad2 and the nad5 genes use ATT and GTG start codons, respectively (Additional file 3: Table S2). The most represented stop codon is TAA, except for the nad3 gene, with TAG, and cox3, nad5 and nad4 genes that have an incomplete TAstop codon (Additional file 3: Table S2). The Lepidurus taxa have similar PCG features: seven genes use the ATG start codon, followed by the ATA $(n=5)$ and a 
Table 2 General information and nucleotide features of newly sequenced mitogenomes and of those drawn from Genbank

\begin{tabular}{|c|c|c|c|c|c|c|c|}
\hline \multirow[t]{2}{*}{ Species } & \multirow[t]{2}{*}{ Length (bp) } & \multirow[t]{2}{*}{ Mean coverage } & \multicolumn{5}{|c|}{ GC-richness } \\
\hline & & & Total & PCGS & rRNAs & tRNAs & Control Region \\
\hline Lepidurus apus lubbocki & 15,635 & $234 x$ & 27.8 & 28.6 & 27.2 & 28.5 & 15.7 \\
\hline L. arcticus & 15,223 & $200 x$ & 32.5 & 33.8 & 27.9 & 29.8 & 27.5 \\
\hline Triops cancriformis (Italy) & 15,158 & $234 x$ & 31.3 & 31.4 & 29.1 & 31.5 & 29.1 \\
\hline T. cancriformis (Spain) & 15,103 & $295 \times$ & 31.3 & 31.4 & 29.1 & 31.5 & 29.1 \\
\hline T. cancriformis (Japan) & 15,101 & - & 31.3 & 31.3 & 29.6 & 31.5 & 28.1 \\
\hline T. australiensis & 15,125 & - & 28.4 & 28.5 & 27.3 & 32.1 & 25.4 \\
\hline T. longicaudatus 1 & 15,110 & - & 30.7 & 31.2 & 28.5 & 30.3 & 26.7 \\
\hline T. longicaudatus 2 & 15,115 & - & 30.6 & 31.1 & 28.9 & 30.1 & 26.8 \\
\hline T. longicaudatus "I" & 15,047 & - & 30.1 & 30.4 & 28.3 & 30.5 & 26.4 \\
\hline T. longicaudatus "s" & 15,028 & - & 30.5 & 31.0 & 28.6 & 30.7 & 27.5 \\
\hline T. newberry & 14,976 & - & 30.8 & 31.4 & 28.6 & 30.6 & 27.5 \\
\hline Artemia franciscana & 15,822 & - & 35.6 & 35.9 & 37.3 & 34.8 & 31.9 \\
\hline A. tibetiana 1 & 15,826 & - & 37.3 & 37.6 & 38.7 & 35.4 & 34.5 \\
\hline A. tibetiana 2 & 15,742 & - & 37.3 & 37.8 & 38.6 & 35.4 & 34.6 \\
\hline A. urmiana & 15,945 & - & 37.5 & 38.2 & 38.0 & 35.5 & 36.0 \\
\hline Phallocryptus tserensodnomi & 16,493 & - & 34.6 & 36.2 & 33.2 & 33.9 & 29.4 \\
\hline Streptocephalus sirindhornae & 16,887 & - & 35.4 & 35.6 & 33.4 & 32.5 & 37.4 \\
\hline Limnadia lenticularis & 15,151 & - & 35.0 & 35.8 & 31.5 & 34.7 & 29.4 \\
\hline Diaphanosoma dubium & 16,353 & - & 34.4 & 34.9 & 32.5 & 34.5 & 41.9 \\
\hline Daphnia carinata & 15,245 & - & 29.7 & 30.1 & 27.9 & 29.6 & 27.4 \\
\hline D. galeata & 16,160 & - & 36.3 & 38.2 & 31.7 & 34.0 & 32.0 \\
\hline D. magna & 14,948 & - & 32.9 & 33.7 & 29.8 & 31.2 & 26.3 \\
\hline D. pulex & 15,333 & - & 37.8 & 39.9 & 32.3 & 34.4 & 32.9 \\
\hline D. pulex (China) & 15,306 & - & 35.5 & 36.7 & 31.6 & 33.8 & 34.6 \\
\hline
\end{tabular}

TGA start codon (nad1 gene) (Additional file 3: Table S2). Even in this instance, TAA is the most used stop codon; only two genes use a different stop codon: nad4, using the incomplete TA- codon, and nad5, using the TAG codon (Additional file 3: Table S1). The GC richness of PCGs varies from $28.6 \%$ ( $L$. apus lubbocki) to $33.8 \%$ (L. arcticus) (Table 2).

The analysis of relative synonymous codon usage (RSCU) among the two Lepidurus taxa and T. cancriformis did not demonstrate substantial differences in codon usage, except for the leucine-encoding TTA in L. apus lubbocki (Additional file 4: Figure S1). All codons were present in PCGs of the examined mitogenomes, except the AGG codon that is absent in L. apus lubbocki and T. cancriformis taxa, and poorly represented in $L$. arcticus (Additional file 4: Figure S1).

The two rRNA genes, $r r n L$ and $r r n S$, are encoded on the $\mathrm{L}$ strand and have GC content $<30.0 \%$ in all samples (Table 2). The length of $r r n S$ is the same in the two Lepidurus taxa, $763 \mathrm{bp}$, and a little shorter in T. cancriformis, $756 \mathrm{bp}$. The $r r n L$ gene, though, shows length variation also between $L$. apus lubbocki and $L$. arcticus, being $3 \mathrm{bp}$ shorter in the latter species. Triops cancriformis $r r n L$ gene is slightly shorter than the Lepidurus ones (Additional file 3: Table S2).

The tRNA gene lengths span between $63 \mathrm{bp}$ and $73 \mathrm{bp}$, with an average GC richness between $28.5 \%$ (L. apus lubbocki) and 31.5\% (T. cancriformis) (Table 2; Additional file 3: Table S2). All tRNAs show the typical cloverleaf structure, except the trnS1 that lacks the D-arm; this is common among metazoan serine-tRNAs [59].

The control-region length in Lepidurus taxa varies between $545 \mathrm{bp}$ (L. arcticus) and $885 \mathrm{bp}$ (L. apus lubbocki), mainly owing to a composite microsatellite motif (CTAT TTAT $)_{22}(\text { TTTA })_{18}(\text { CTATTTAT })_{9}(\text { TTTA })_{15}$ in L. apus lubbocki. This repeat motif, moreover, appears to be responsible for the AT richness of this region (Table 2): in fact, if excluded from the analysis, the GC content rises to $21.2 \%$. The length of the control region in the T. cancriformis samples is 467 bp (Spain) and 522 bp (Italy), the difference being due to a $55 \mathrm{bp}$ duplication at the $5^{\prime}$ end of the latter.

Within the newly sequenced mitogenomes there are few intergenic regions, spanning from 1 to 22 nucleotides. On 


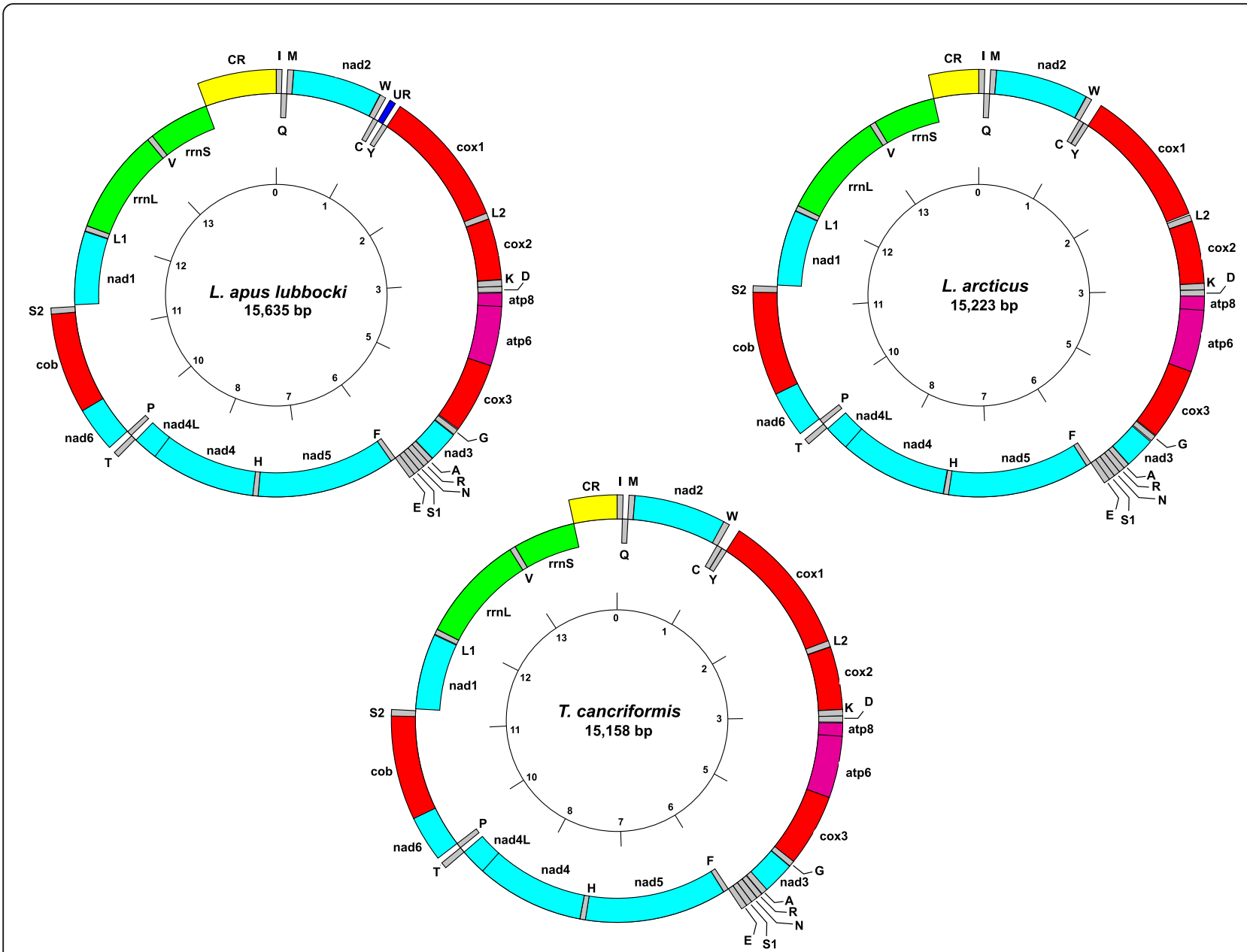

Fig. 1 Schematic structures of newly sequenced mitochondrial genomes

the other hand, a larger unassigned region (UR) of $69 \mathrm{bp}$ can be observed in the L. apus lubbocki mitogenome, located between the $\operatorname{trn} C$ and $\operatorname{trn} Y$ genes (Fig. 1; Additional file 3: Table S2). A visual inspection reveals that the UR is a chimeric sequence between the two flanking tRNAs; moreover, a high similarity region $(82.6 \%)$ is shared between $\operatorname{trn} C$ and $\operatorname{trn} Y$ sequences, corresponding to the anti-codon arm (Fig. 2a). In order to determine whether the chimeric structure derives from an assembly error, reads were mapped on the region spanning the $\operatorname{trn} C+$ UR + trnY: this showed a maximum coverage of $214 \times$, with 109 reads covering entirely the whole UR (Fig. 2b), allowing us to rule out the possibility of an assembly artefact. Alternatively, it can be hypothesized that the assembled UR may have included reads from a nuclear mitochondrial DNA segment (NUMT) [60]. NUMTs are usually a serious issue in PCR-based approaches, because of preferential PCR amplification due to possible biases in primer annealing on NUMT sequence rather than on true mitochondrial DNA. However, this should not be an issue in whole genome sequencing. In addition, because of their high cellular copy number, mitochondrial reads are highly represented in genome sequencing, greatly outnumbering those of NUMTs [61]. Furthermore, NUMTs are pseudogenic sequences that can be typically distinguished by the accumulation of substitutions and indels with respect to genuine mitochondrial sequences [60]; therefore, we would have seen different pools of reads mapping to either the NUMT or the mitochondrial genome. Thus, we checked for the possible presence of a mtDNA variant without the UR and showing the structure expected from other notostracan mitogenomes. We manually produced a sequence which included only the $\operatorname{trn} C$ and $\operatorname{trn} Y$ genes and used it to map reads: this resulted in no read mapping, thus excluding the possibility of having missed the ancestral variant and making unlikely that we mistakenly assembled a NUMT in the L. apus lubbocki mitogenome.

Overall, these results suggest the UR could be the product of a non-homologous recombination. It is, in fact, possible that two mitochondrial molecules misaligned due to the similarity of the anti-codon arm domain and an unequal DNA exchange occurred (Fig. 2c). 


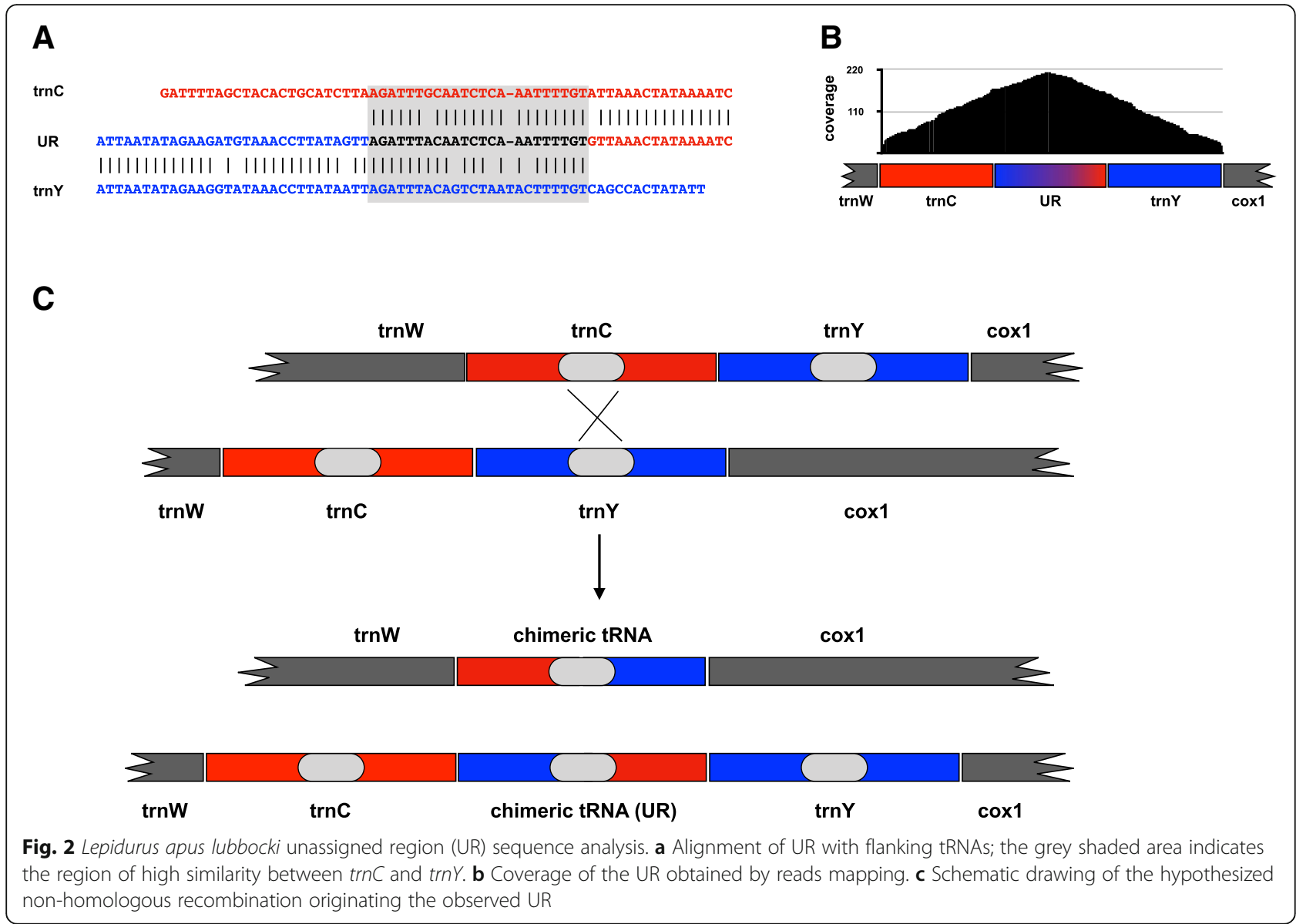

Then, two different haplotypes were produced: one including only a chimeric tRNA but not the $\operatorname{trn} C$ and $\operatorname{trn} Y$ genes, the other including a chimeric tRNA (the actual UR) and the two flanking tRNA genes (Fig. 2c). In order to check whether sequences including only the chimeric tRNA were present but not assembled, we manually created this sequence, and found that no reads map to it. We can conclude that the variant carrying only the chimeric tRNA is not present in the sequenced individual; since this variant would lack two tRNA genes, it is likely that it has been lost due to natural selection.

Homologous and non-homologous mitochondrial DNA recombination has been reported in animal mitochondria, both in vitro or in vivo [62]; in particular, evidence of non-homologous recombination has been reported in the control region of the root-knot nematode Meloidogyne javanica [63]. To our knowledge, data presented here constitute the first evidence of a possible non-homologous recombination event within the genic part of the mitochondrial genome. It is interesting to speculate about the fate of the observed UR. Although originating from the recombination of two tRNA genes, the observed UR was not recognized as a tRNA by the annotation software and it does not fold into a proper cloverleaf structure. Following the secondary structures of the flanking $\operatorname{trn} C$ and $\operatorname{trn} Y$, we sought to reconstruct a hypothetical secondary structure for the UR (Additional file 4: Figure S2). While generally consistent with the typical cloverleaf structure, with a conserved tyrosine anticodon, lack of complementarity between the two strands of the acceptor arm seems to preclude its functionality. It is possible that point mutations occurring in this domain could stabilize the structure, which opens the possibility of recruiting the UR as a new copy of $\operatorname{trn} Y$ gene.

\section{Gene order variability among Branchiopoda}

With the exception of the UR in the L. apus lubbocki, notostracan mitogenomes share the same gene order, consistent with the ancestral Pancrustacea model (Fig. 3) . Within Onychocaudata, the same holds also for Limnadia lenticularis, and most of Daphnia species. In the Daphnia magna mitogenome, on the other hand, the trnI gene and the control region experienced an inversion. The most rearranged mitogenome, actually, is that of Diaphanosoma dubium showing extensive rearrangement of tRNA genes. Moreover, it showed a 978 bp long UR, upstream the cox 1 gene: this appears to have a coding potential but the resulting protein (325 aa) does not 


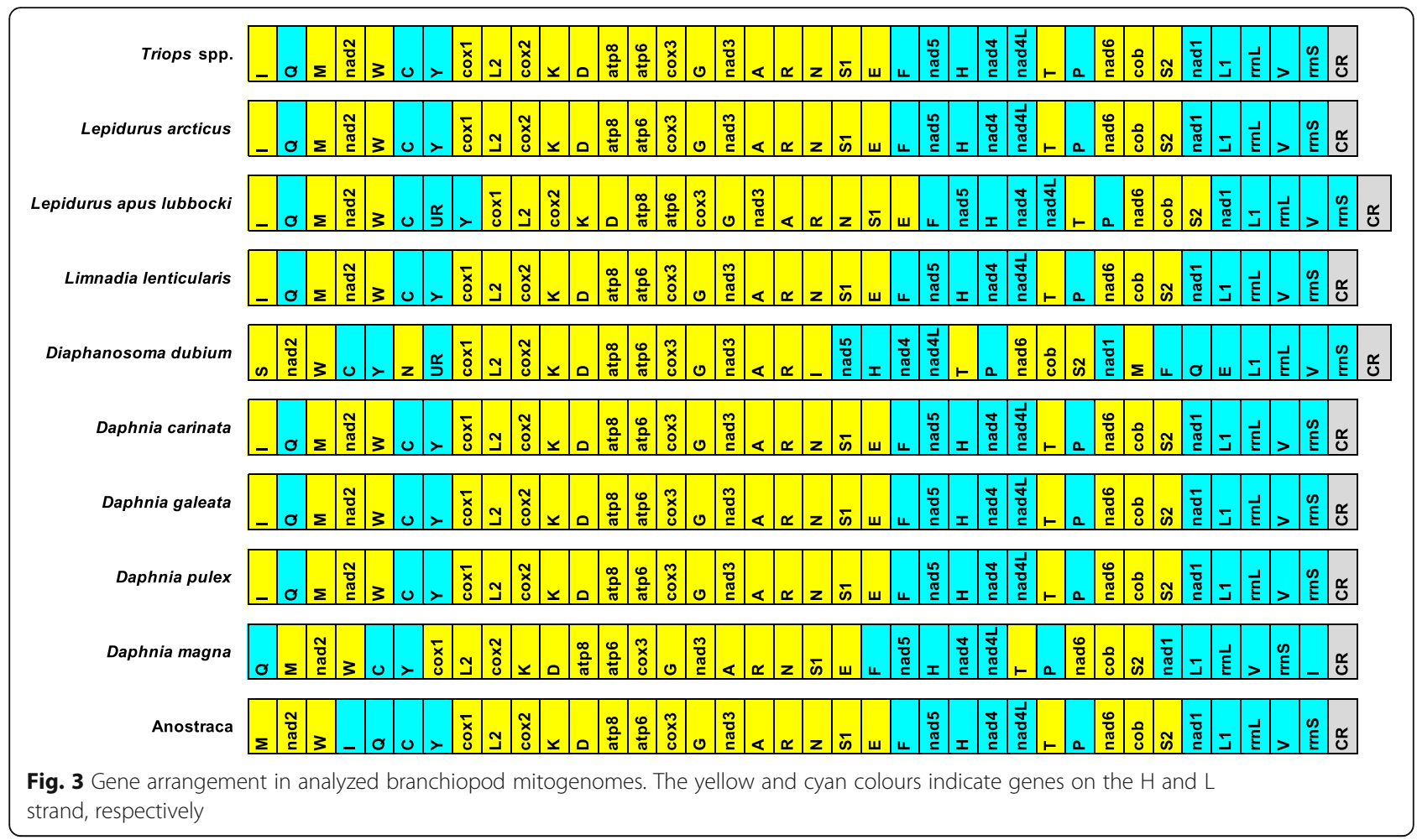

match any known protein (not shown) [40]. Anostracan mitogenome does not vary among the five species analyzed, although it showed a tRNA rearrangement with respect to the ancestral Pancrustacea model (Fig. 3). Generally speaking, therefore, branchiopod mitogenomes appear well conserved, although showing a certain degree of structural variability.

\section{Nucleotide variability and phylogenetic analyses}

The nucleotide divergence within genera observed among PCGs and rRNAs of newly sequenced mitogenomes ranged from $0.18 \%$, in a comparison between
Italian and Spanish T. cancriformis, to $20.25 \%$, as scored between the two Lepidurus taxa. Moreover, with respect to the Japanese T. cancriformis, the Italian and Spanish T. cancriformis sequences diverge by 0.24 and $0.32 \%$, respectively (Table 3 ). These estimates are in line with previous mitochondrial survey between Triops cancriformis populations and between Lepidurus species [22, 24]. An overall evaluation among Notostraca (Additional file 4: Figure S3) revealed that the least variable genes are the rRNAs (divergence $r r n L=17.3 \%$ and $r r n S=17.5$ ), while the most variable ones resulted the nad6 (37.6\%) and the atp 8 (37.9\%). On average, rRNA genes are the less

Table 3 Sequence divergence (\%) between Notostraca taxa based on PCG and rRNA gene sequences. Species with newly sequenced mitogenomes are marked in bold

\begin{tabular}{|c|c|c|c|c|c|c|c|c|c|c|}
\hline & 1 & 2 & 3 & 4 & 5 & 6 & 7 & 8 & 9 & 10 \\
\hline \multicolumn{11}{|l|}{ 1. L. arcticus } \\
\hline 2. L. apus lubbocki & 20.25 & & & & & & & & & \\
\hline 3. T. cancriformis (Italy) & 26.77 & 26.13 & & & & & & & & \\
\hline 4. T. cancriformis (Spain) & 26.80 & 26.19 & 0.18 & & & & & & & \\
\hline 5. T. cancriformis (Japan) & 26.78 & 26.17 & 0.24 & 0.32 & & & & & & \\
\hline 6. T. longicaudatus "I" & 26.16 & 25.66 & 22.06 & 22.11 & 22.09 & & & & & \\
\hline 7. T. longicaudatus "s" & 26.03 & 25.55 & 22.04 & 22.09 & 22.06 & 3.84 & & & & \\
\hline 8. T. longicaudatus 1 & 26.23 & 25.74 & 22.26 & 22.30 & 22.25 & 4.08 & 2.04 & & & \\
\hline 9. T. longicaudatus 2 & 26.12 & 25.69 & 22.14 & 22.16 & 22.14 & 4.16 & 1.73 & 2.54 & & \\
\hline 10. T. newberry & 26.12 & 25.66 & 22.03 & 22.08 & 22.03 & 3.75 & 1.83 & 0.72 & 2.17 & \\
\hline 11. T. australiensis & 25.50 & 24.73 & 22.02 & 22.08 & 22.03 & 15.37 & 15.39 & 15.57 & 15.29 & 15.33 \\
\hline
\end{tabular}


variable and the genes encoding for NADH subunits are the most variable. This trend is maintained when all available branchiopod mitogenomes $(N=24)$ are considered, with the only difference that the least variable gene is $\operatorname{cox} 1$ (Additional file 4: Figure S3).

Phylogenetic analyses were conducted on concatenated PCG and rRNA genes. The whole dataset consisted of 14, 274 aligned nucleotide positions, with 10,214 variable and 9266 parsimony informative sites. Both maximum-likelihood analysis and the Bayesian inference gave a topology (Fig. 4) in agreement with the current knowledge on branchiopod phylogeny $[4,6-8]$. The deepest node splits the two clusters representing the Anostraca and the Phyllopoda clades. The two groups are well supported in the Bayesian inference, showing posterior probability equals to 1.0 and 0.99 , respectively, but in the maximum-likelihood tree the Phyllopoda clade is only weakly supported (bootstrap $=55 \%$ ). Within Anostraca, the split between suborders Artemiina (Artemia spp.) and Anostracina (Phallocryptus + Streptocephalus) had the maximum support in both elaborations. Within Phyllopoda, Onychocaudata (Limnadia lenticularis + cladoceran taxa) received maximum nodal support only in the Bayesian inference, while Notostraca is fully supported in all analyses. Relationships within Notostraca fully agree with previous

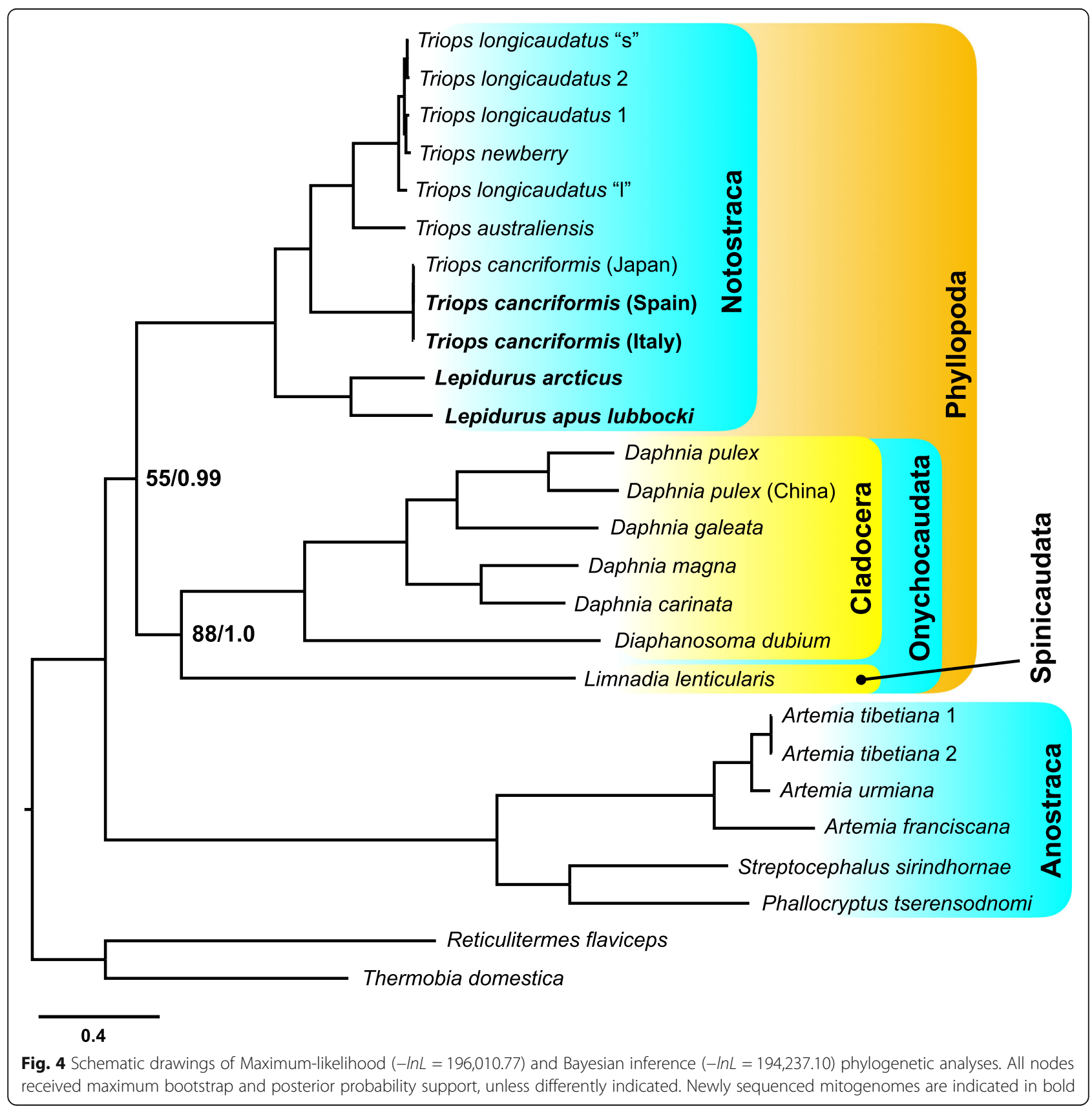


analyses: the two Lepidurus sequences cluster together and are in sister relationship with the Triops clade $[64,65]$.

\section{Nucleotide compositional bias among Branchiopoda}

Branchiopod mitogenomes are generally AT-rich, the GC content varying between $28.2 \%$ in T. australiensis to $38.9 \%$ in D. pulex (Table 2). In the phylogenetic dataset, the average GC content was $30.5 \%$ in Notostraca, $35.0 \%$ in Onychocaudata and $36.8 \%$ in Anostraca, and results significantly different between clades (Kruskal-Wallis $P<0.001$ ). Pairwise comparisons indicate that Notostraca have a significantly lower GC content than Anostraca or Onychocaudata (Mann-Whitney pairwise post-hoc test, with sequential Bonferroni correction, $P<0.01$ ), while the latter two clades do not differ significantly (Mann-Whitney posthoc test $P=0.197$ ). In order to determine whether nucleotide composition has varied since the common ancestor, which would mean a shift in nucleotide composition during the evolution of the three clades, we reconstructed the ancestral sequences at stem nodes of each of the three orders and compared their GC content to that of extant lineages. To avoid possible biases in the reconstruction process we followed the recommendation in Matsumoto et al. [56], sampling multiple sequences from the posterior distribution ( $n=100$; Additional files 1 and 2) and calculated the average GC content. Average GC content of ancestral sequences sampled from the posterior distribution at Anostraca stem node is $31.0 \%$, very close to the $30.5 \%$ calculated at Onychocaudata and Notostraca stem nodes. Overall, GC content varies significantly among ancestral nodes and extant lineages (Kruskal-Wallis $P<0.001$ ); however, while Notostraca do not show a significant departure from ancestral GC content (Mann-Whitney post-hoc test $P=$ 0.249), Anostraca and Onychocaudata sequences show a significant increase of GC richness from their relative stem node (Mann-Whitney post-hoc test $P<0.001$ and $P<0.01$, respectively). Therefore, the observed pattern of nucleotide composition in Branchiopoda can be explained by a preferential AT to GC substitution bias during the evolution of Anostraca and Onychocaudata lineages. Differential GC contents and inferred substitution bias do not appear to have had substantial effects on phylogenetic reconstructions, although it cannot be excluded that the weak support at Phyllopoda clade could be due to similar GC content between Anostraca and Onychocaudata mitogenomes.

\section{Differential substitution rates between clades}

In all phylogenetic elaborations, the branches of Onychocaudata and Anostraca clades, including stem branches, appeared longer than those of the Notostraca clade (Fig. $4)$. Interestingly, the same pattern can be observed in a recently published phylogenomic study ( $>600$ orthologues) [8]. Substitution rate constancy throughout the tree was rejected (likelihood-ratio test, $\Delta_{\operatorname{lnL}}=55,050,314.81 ; P<$
0.0001), and the two-cluster test analysis (Additional file 3: Table S3) clearly indicates that, with respect to the Phyllopoda cluster, Anostraca show a significantly higher substitution rate $(\Delta=0.0462 \pm 0.0018 ; Z=25.42$, confidence probability $=99.96 \%)$. Furthermore, Onychocaudata show a higher substitution rate with respect to Notostraca $(\Delta=$ $0.0258 \pm 0.0011 ; \quad Z=22.18$, confidence probability $=$ 99.96\%). Therefore, these data clearly support a differential substitution rate of Notostraca with respect to the Onychocaudata and Anostraca clades.

In order to calculate the substitution rate per branch, a calibrated time tree was built by dating Notostraca, Cladocera and Anostraca crown groups, along with the tree root, using well-detailed and justified fossil records described in Wolfe et al. [54] for points calibration. The obtained tree topology resulted identical to those produced with maximum-likelihood and Bayesian inference elaborations, with all nodes receiving maximum posterior probability support (Fig. 5). Inferred age estimates reconcile well with those obtained in previous analyses $[64,65]$ : diversification of Branchiopoda occurred since 425.7 Mya and extant lineages of Notostraca, Cladocera, and Anostraca (crown groups) radiated between 243.0 Mya and 143.9 Mya. The estimated mean substitution rate is $3.21 \times 10^{-3}$ substitutions/site/million year $(95 \%$ high posterior density interval $=2.81 \times 10^{-3}, 3.55 \times 10^{-3}$ ) . This rate varies among branches between $1.88 \times 10^{-3}$ substitutions/site/million year and $5.24 \times 10^{-3}$ substitutions/site/million year, the two values being estimated for Notostraca and Anostraca stem branches, respectively. Overall, it seems that substantial rate variation occurred along the stem branches of analyzed clades (Fig. 5; Additional file 4: Figure S4). The lower substitution rate observed along the Notostraca stem branch, 2.7-fold and 1.7-fold with respect to Anostraca and Diplostraca (1.9-fold vs Cladocera), respectively, could be either interpreted as i) an episodic substitution rate decrease along the Notostraca stem branch, or ii) an acceleration of the substitution rate in the other stem lineages with respect to the notostracan one.

These data are in agreement with those from a recent genome-wide survey where the nucleotide substitution rate calculated for L. arcticus and L. apus lubbocki was found to be significantly lower than the one calculated for D. magna and D. pulex. In contrast to this, however, the amino acid substitution rate did not vary significantly, in line with the observed general pattern of negative selection [25]. We therefore checked the extent of selective pressures on mitogenomic sequences calculating the $\omega$ ratio across the tree branches. Two different models were run: the free-ratio model, allowing an $\omega$ value for each branch and the one-ratio model, considering a single ratio for the whole tree. The free-ratio model resulted the best fit one (likelihood-ratio test, $\Delta_{\operatorname{lnL}}=608.002, P<0.001$ ). No 


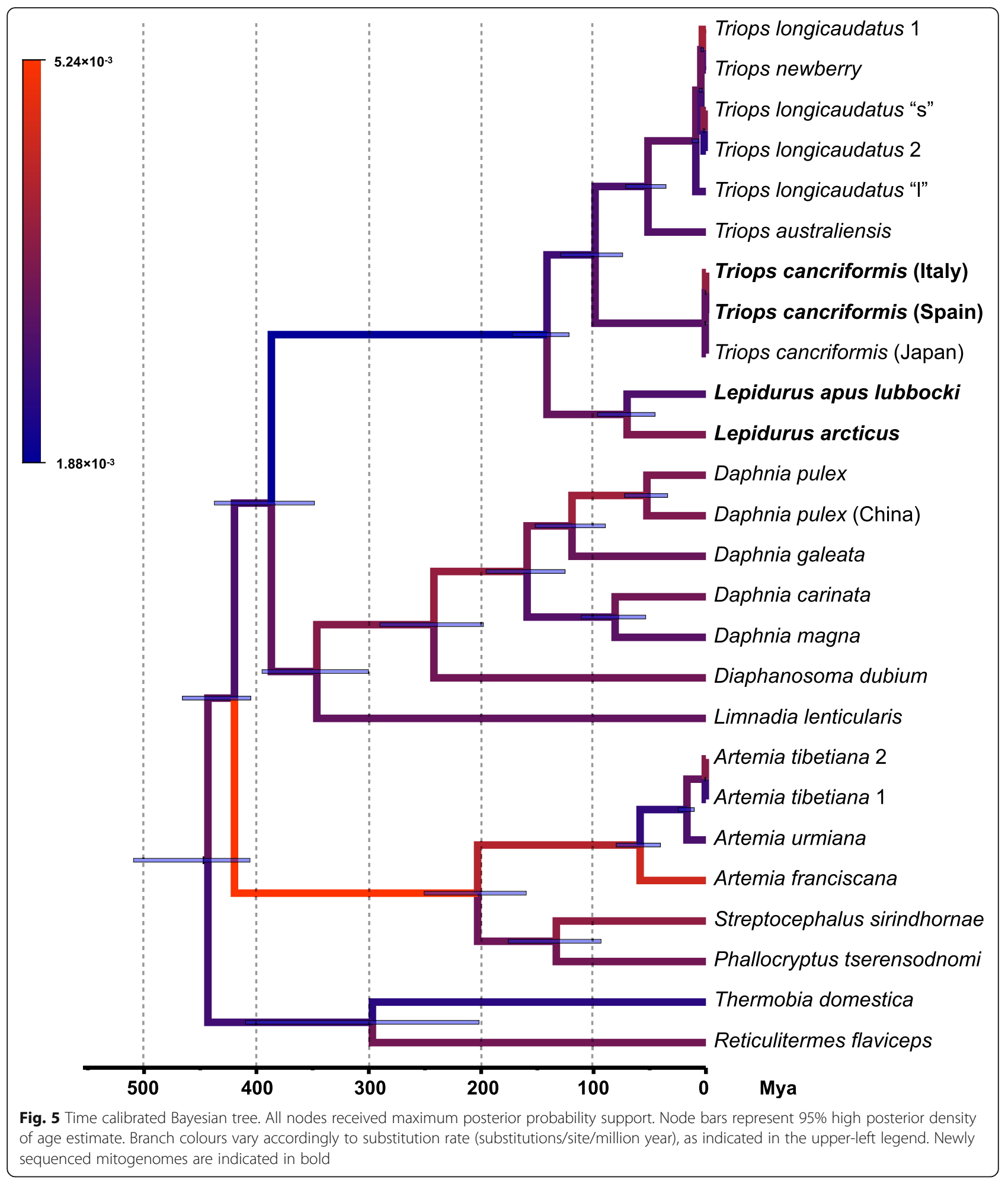

instances of positive selection, $\omega>1$, have been found; the highest $\omega$ values were scored on the Phyllopoda (0.493) and Onychocaudata (0.402) stem branches, possibly suggesting a relaxation of natural selection on these branches. Interestingly, both Anostraca and
Notostraca stem branches show decidedly lower $\omega$ values ( 0.127 and 0.184 , respectively), consistent with purifying selection. Overall, the present data do not support any correlation between the observed substitution rates variation and changes in natural 
selection regime, in agreement with genome-wide data [25].

Variation in substitution rates could depend not only on life-history traits, such as generation time, lifespan, and body size, but also on speciation rate [66-70]. In Branchiopoda, it has also been suggested that halophilic habits may correlate with faster substitution rates [71]. Although these hypotheses cannot be reliably tested with our species sampling, an alternative explanation could be linked to the nucleotide substitution bias observed among the three branchiopod orders. Based on mathematical models and simulations, Sueoka [72] showed that a directional mutation bias during lineages' evolution may significantly affect substitution rates. Accordingly, an episodic acceleration of substitution rate along the Diptera stem branch has been observed and found consistent with directional mutational bias during the evolution of this taxon [73]. Based on the conclusions of these studies, the observed GC-biased mutation rate would speak in favour of an increase of the substitution rate in Anostraca and Onychocaudata rather than an episodic deceleration in Notostraca, whose GC content appeared similar to that of the ancestor. Moreover, the Sueoka's model [72] also predicts that changes in nucleotide composition would result in asymmetrical branch length in the phylogenetic tree, which fits with the phylogenetic tree obtained in our analysis.

\section{Conclusions}

In the present study, we present four new Notostraca mitochondrial genomes, with two of them reported for the first time. We also carried out a comparative analysis with other available branchiopod mitogenomes. The analysis of mitogenomes' gene order revealed a certain plasticity within and among Branchiopoda orders, and with respect to the ancestral Pancrustacea model. Notably, we detected the results of a possible unequal recombination in the $L$. apus lubbocki mitogenome which led to the formation of a pseudogenic tRNA variant. This is, to our knowledge, the first example of an unequal recombination event involving mitochondrial genes. Overall, the phylogenetic signal contained in the sequences appears reliable despite significant differences in substitution rate and nucleotide composition. The tree topologies here obtained confirm those previously obtained on fewer molecular markers or on phylogenomic datasets. In addition, we showed differential substitution rates among Branchiopoda orders; these could be likely linked to changes in nucleotide composition during evolution. As genomic data are accumulating, and rate differences have been observed also at the nuclear genomic level [25], it will be interesting to test this observation on a wider phylogenomic dataset.

\section{Additional files}

Additional file 1: 100 ancestral sequences sampled from the posterior distribution of Anostraca stem node (FASTA $1394 \mathrm{~kb}$ )

Additional file 2: 100 ancestral sequences sampled from the posterior distribution of Phyllopoda stem node (FASTA $1394 \mathrm{~kb}$ )

Additional file 3: Supplementary Tables S1, S2, S3 (XLSX 18 kb)

Additional file 4: Supplementary Figures S1, S2, S3, S4 (PDF 218 kb)

\section{Acknowledgements}

We wish to thank Fabrizio Ghiselli and Federico Plazzi for critical reading of the manuscript and for fruitful discussions. We are grateful to the Editor and Reviewers for their comments and suggestions that greatly helped in improving the manuscript.

\section{Authors' contributions}

AL conceived the study, carried out DNA isolation, mitogenome assemblies, performed comparative and phylogenetic analyses, wrote the paper; GF carried out selection analyses and helped in phylogenetic analyses; AMS carried out Triops sequencing and helped in assemblies; SJW and BM conceived and coordinate the study, helped to write the manuscript. All authors read and approved the final manuscript.

\section{Funding}

The work has been supported by Canziani Funding to BM and by the National Institute of Health/National Cancer Institute grant NIH P30 CA006973 to SJW.

\section{Availability of data and materials}

Newly obtained mitogenomes have submitted to GenBank under accession numbers MK579380-MK579383.

Ethics approval and consent to participate

Not applicable.

Consent for publication

Not applicable.

\section{Competing interests}

The authors declare that they have no competing interests.

\section{Author details}

${ }^{1}$ Department of Biological, Geological and Environmental Sciences, University of Bologna, via Selmi 3, 40126 Bolgna, Italy. ²Department of Oncology, Sidney Kimmel Comprehensive Cancer Center, The Johns Hopkins University School of Medicine, Baltimore, MD 21205, USA.

Received: 13 December 2018 Accepted: 19 May 2019

Published online: 27 May 2019

\section{References}

1. Brendonck L, Rogers DC, Olesen J, Weeks S, Hoeh WR. Global diversity of large branchiopods (Crustacea: Branchiopoda) in freshwater. Hydrobiologia. 2008;595:167-76.

2. Forró L, Korovchinsky NM, Kotov AA, Petrusek A. Global diversity of cladocerans (Cladocera; Crustacea) in freshwater. Hydrobiologia. 2008;595:177-84.

3. Rogers DC. Branchiopoda (Anostraca, Notostraca, Laevicaudata, Spinicaudata, Cyclestherida). In: Likens GE, editor. Encyclopedia of inland waters, vol. 2. Oxford: Elsevier Sceince; 2009. p. 242-9.

4. Olesen J. Phylogeny of Branchiopoda (Crustacea) - character evolution and contribution of uniquely preserved fossils. Arthropod Syst Phylo. 2009;67:3-39.

5. Sun X-Y, Xia X, Yabg Q. Dating the origin of the major lineages of Branchiopoda. Palaeoworld. 2016;25:303-17.

6. von Reumont BM, Jenner RA, Wills MA, Dell'Ampio E, Pass G, Ebersberger I, Meyer B, Koenemann S, Iliffe TM, Stamatakis A, Niehuis O, Meusemann K, 
Misof B. Pancrustacean phylogeny in the light of new phylogenomic data: support for Remipedia as the possible sister group of Hexapoda. Mol Biol Evol. 2012;29:1031-45.

7. Schwentner M, Combosch DJ, Nelson JP. Giribet. A phylogenomic solution to the origin of insects by resolving crustacean-hexapod relationships. Curr Biol. 2017:27:1818-24.

8. Schwentner M, Richter S, Rogers DC, Giribet G. Tetraconatan phylogeny with special focus on Malacostraca and Branchiopoda: highlighting the strength of taxon-specific matrices in phylogenomics. Proc R Soc B. 2018; 285:20181524

9. Smith DR. The past, present and future of mitochondrial genomics: have we sequenced enough mtDNAs? Brief Funct Genomics. 2015;15:47-54.

10. Boore JL. Animal mitochondrial genomes. Nuc Acids Res. 1999;27:1767-80.

11. Bernt M, Braband A, Middendorf M, Misof B, Rota-Stabelli O, Stadler PF. Bioinformatics methods for the comparative analysis of metazoan mitochondrial genome sequences. Mol Phylogenet Evol. 2013;69:320-7.

12. Lavrov DV, Pett W. Animal mitochondrial DNA as we do not know it: mtgenome organization and evolution in nonbilaterian lineages. Genome Biol Evol. 2016:8:2896-913.

13. Gissi C, lannelli F, Pesole G. Evolution of the mitochondrial genome of Metazoa as exemplified by comparison of congeneric species. Heredity. 2008; 101:301-20

14. Guerra D, Bouvet K, Breton S. Mitochondrial gene order evolution in Mollusca: inference of the ancestral state from the mtDNA of Chaetopleura apiculata (Polyplacophora, Chaetopleuridae). Mol Phylogenet Evol. 2018;120:233-9.

15. Stokkan M, Jurado-Rivera JA, Juan C, Jaume D, Pons J. Mitochondrial genome rearrangements at low taxonomic levels: three distinct mitogenome gene orders in the genus Pseudoniphargus (Crustacea: Amphipoda). Mitochondrial DNA. 2016;27:3579-89.

16. Basso A, Babbucci M, Pauletto M, Riginella E, Patarnello T, Negrisolo E. The highly rearranged mitochondrial genomes of the crabs Maja crispata and Maja squinado (Majidae) and gene order evolution in Brachyura. Sci Rep. 2017;7:4096

17. Liu H, Li H, Song F, Gu W, Feng J, Cai W, Shao R. Novel insights into mitochondrial gene rearrangement in thrips (Insecta: Thysanoptera) from the grass thrips, Anaphothrips obscurus. Sci Rep. 2017;7:4284.

18. Jiang P, Li H, Song F, Cai Y, Wang J, Liu J, Cai W. Duplication and remolding of tRNA genes in the mitochondrial genome of Reduvius tenebrosus (Hemiptera: Reduviidae). Int J Mol Sci. 2016;17:951.

19. Song F, Li H, Shao R, Shi A, Bai X, Zheng X, Heiss E, Cai W. Rearrangement of mitochondrial tRNA genes in flat bugs (Hemiptera: Aradidae). Sci Rep. 2016;6:25725

20. Ye F, X-e L, W-b Z, You P. Mitochondrial genomes of praying mantises (Dictyoptera, Mantodea): rearrangement, duplication, and reassignment of tRNA genes. Sci Rep. 2016;6:25634.

21. Jühling F, Pütz J, Bernt M, Donath A, Middendorf M, Florentz C, Stadler PF. Improved systematic tRNA gene annotation allows new insights into the evolution of mitochondrial tRNA structures and into the mechanisms of mitochondrial genome rearrangements. Nuc Acids Res. 2012;40:2833-45.

22. Mantovani B, Cesari M, Scanabissi F. Molecular taxonomy and phylogeny of Italian Lepidurus taxa (Branchiopoda: Notostraca). It J Zool. 2009;76:358-65.

23. Umetsu K, Iwabuchi N, Yuasa I, Saitou N, Clark PF, Boxshall G, Osawa M, Igarashi K. Complete mitochondrial DNA sequence of a tadpole shrimp (Triops cancriformis) and analysis of museum samples. Electrophoresis. 2002; 23:4080-4.

24. Mantovani B, Cesari M, Luchetti A, Scanabissi F. Mitochondrial and nuclear DNA variability in the living fossil Triops cancriformis (bosc, 1801) (Crustacea, Branchiopoda, Notostraca). Heredity. 2008;100:496-505.

25. Savojardo C, Luchetti A, Martelli PL, Casadio R, Mantovani B. Draft genomes and genomic divergence of two Lepidurus tadpole shrimp species (Crustacea, Branchiopoda, Notostraca). Mol Ecol Resour. 2019;19:235-44.

26. Nurk S, Bankevich A, Antipov D, Gurevich A, Korobeynikov A, Lapidus A, et al. Assembling genomes and mini-metagenomes from highly chimeric reads. In: Deng $M$, Jiang $R$, Sun $F$, Zhang $X$, editors. Research in computational molecular biology. RECOMB 2013. Lecture notes in computer science, vol. 7821. Berlin, Heidelberg: Springer; 2013. p. 158-70.

27. Langmead B, Salzberg SL. Fast gapped-read alignment with bowtie 2. Nat Methods. 2012;9:357-9.

28. Li H. A statistical framework for SNP calling, mutation discovery, association mapping and population genetical parameter estimation from sequencing data. Bioinformatics. 2011;27:2987-93.
29. Bernt M, Donath A, Jühling F, Externbrink F, Florentz C, Fritzsch G, Pütz J, Middendorf M, Stadler PF. MITOS: improved de novo metazoan mitochondrial genome annotation. Mol Phylogenet Evol. 2013;69:313-9.

30. Laslett $D$, Canbäck B. ARWEN, a program to detect tRNA genes in metazoan mitochondrial nucleotide sequences. Bioinformatics. 2008;24:172-5.

31. Perez ML, Valverde JR, Batuecas B, Amat F, Marco R, Garesse R. Speciation in the Artemia genus: mitochondrial DNA analysis of bisexual and parthenogenetic brine shrimps. J Mol Evol. 1994;38:156-68.

32. Zhang H, Luo Q, Sun J, Liu F, Wu G, Yu J, Wang W. Mitochondrial genome sequences of Artemia tibetiana and Artemia urmiana: assessing molecular changes for high plateau adaptation. Sci China Life Sci. 2013;56:440-52.

33. Fan Y-P, Lu B, Yang J-S. The complete mitogenome of the fairy shrimp Phallocryptus tserensodnomi (Crustacea: Anostraca: Thamnocephalidae). Mitochondrial DNA Part A. 2016;27:3113-4.

34. Liu X-C, Li H-W, Jermnak U, Yang J-S. The complete mitogenome of the freshwater fairy shrimp Streptocephalus sirindhornae (Crustacea: Anostraca: Streptocephalidae). Mitochondrial DNA Part A. 2016;27:3189-91.

35. Geng X, Cheng R, Deng D, Zhang H. The complete mitochondrial DNA genome of Chinese Daphnia carinata (Cladocera: Daphniidae). Mitochondrial DNA Part B. 2016;1:323-5.

36. Cheng R, Deng B, Wang Y, Geng X, Li J, Zhang X, Peng S, Deng D, Zhang H. Complete mitochondrial genome sequence of Daphnia magna (Crustacea: Cladocera) from Huaihe in China. J Lake Sci. 2016;28:414-20.

37. Crease TJ. The complete sequence of the mitochondrial genome of Daphnia pulex (Cladocera: Crustacea). Gene. 1999;233:89-99.

38. Geng X, Cheng R, Xiang T, Deng B, Wang Y, Deng D, Zhang H. The complete mitochondrial genome of the Chinese Daphnia pulex (Cladocera, Daphniidae). Zookeys. 2016;615:47-60.

39. Tokishita SI, Shibuya H, Kobayashi T, Sakamoto M, Ha JY, Yokobori SI, Yamagata $\mathrm{H}$, Hanazat T. Diversification of mitochondrial genome of Daphnia galeata (Cladocera, Crustacea): comparison with phylogenetic consideration of the complete sequences of clones isolated from five lakes in Japan. Gene. 2017;611:38-46.

40. Liu P, Xu S, Huang Q, Dumont HJ, Lin Q, Han B-P. The mitochondrial genome of Diaphanosoma dubium with comparison with Daphnia magna. Mitochondrial DNA part B. 2018;2:926-7.

41. Bellec L, Debruyne R, Utge J, Rabet N. The first complete mitochondrial genome of Limnadia lenticularis (Branchiopoda, Spinicaudata), with new insights on its phylogeography and on the taxonomy of the genus. Hydrobiologia. 2019;826:145-58.

42. Gan HM, Tan MH, Lee YP, Austin CM. The complete mitogenome of the Australian tadpole shrimp Triops australiensis (Spencer \& Hall, 1895) (Crustacea: Branchiopoda: Notostraca). Mitochondrial DNA part A. 2016;27:2028-9.

43. Cook CE, Yue Q, Akam M. Mitochondrial genomes suggest that hexapods and crustaceans are mutually paraphyletic. Proc Biol Sci. 2005:272:1295-304.

44. Ryu JS, Hwang UW. Complete mitochondrial genome of the longtail tadpole shrimp Triops Iongicaudatus (Crustacea, Branchiopoda, Notostraca). Mitochondrial DNA. 2010;21:170-2.

45. Horn RL, Cowley DE. Evolutionary relationships within the Triops (Branchiopoda: Notostraca) using complete mitochondrial genomes. J Crust Biol. 2014;34:795-800,

46. Zhao S, Dang YL, Zhang HG, Guo XH, Su XH, Xing LX. The complete mitochondrial genome of the subterranean termite Reticulitermes flaviceps (Isoptera: Rhinotermitidae). Conservation Genet Resour. 2016:8:451-3.

47. Katoh K, Standley DM. MAFFT multiple sequence alignment software version 7: improvements in performance and usability. Mol Biol Evol. 2013;30:772-80.

48. Castresana J. Selection of conserved blocks from multiple alignments for their use in phylogenetic analysis. Mol Biol Evol. 2000;17:540-52.

49. Lanfear R, Calcott B, Ho SYW, Guindon S. PartitionFinder: combined selection of partitioning schemes and substitution models for phylogenetic analyses. Mol Biol Evol. 2012;29:1695-701.

50. Stamatakis A. RAxML version 8: a tool for phylogenetic analysis and postanalysis of large phylogenies. Bioinformatics. 2014;30:1312-3.

51. Ronquist F, Teslenko M, Van Der Mark P, Ayres DL, Darling A, Höhna S, et al. MrBayes 3.2: efficient Bayesian phylogenetic inference and model choice across a large model space. Syst Biol. 2012;61:539-42.

52. Kumar S, Stecher G, Tamura K. MEGA7: molecular evolutionary genetics analysis version 7.0 for bigger datasets. Mol Biol Evol. 2016;33:1870-4.

53. Drummond AJ, Rambaut A. BEAST: Bayesian evolutionary analysis by sampling trees. BMC Evol Biol. 2007;7:214.

54. Wolfe JM, Daley AC, Legg DA, Edgecomb GD. Fossil calibrations for the arthropod tree of life. Earth Sci Rev. 2016;160:43-110. 
55. Ashkenazy H, Penn O, Doron-Faigenboim A, Cohen O, Cannarozzi G, Zomer O, Pupko T. FastML: a web server for probabilistic reconstruction of ancestral sequences. Nuc Acids Res. 2012;40:W580-4.

56. Matsumoto T, Akashi H, Yang Z. Evaluation of ancestral sequence reconstruction methods to infer nonstationary patterns of nucleotide substitution. Genetics. 2015;200:873-90.

57. Takezaki N, Rzhetsky A, Nei M. Phylogenetic tests of the molecular clock and linearized trees. Mol Biol Evol. 1995;12:823-33.

58. Yang Z. PAML 4: a program package for phylogenetic analysis by maximum likelihood. Mol Biol Evol. 2007;24:1586-91.

59. Watanabe Y-I, Suematsu T, Ohtsuki T. Losing the stem-loop structure from metazoan mitochondrial tRNAs and co-evolution of interacting factors. Front Genet. 2014;5:109.

60. Bensasson D, Zhang D-X, Hartl DL, Hewitt GM. Mitochondrial pseudogenes: evolution's misplaced witnesses. Trends Ecol Evol. 2001;16:314-21.

61. Ekblom R, Smeds L, Ellegren H. Patterns of sequencing coverage bias revealed by ultra-deep sequencing of vertebrate mitochondria. BMC Genomics. 2014;15:467.

62. Roaks A, Laudokakis E, Zours E. Animal mitochondrial DNA recombination revisited. Trends Ecol Evol. 2003;18:411-7.

63. Lunt DH, Hyman BC. Animal mitochondrial DNA recombination. Nature. 1997;387:247.

64. Korn M, Rabet N, Ghate HV, Marrone F, Hundsdoerfer AK. Molecular phylogeny of Notostraca. Mol Phylogenet Evol. 2013;69:1159-71.

65. Mathers TC, Hammond RL, Jenner RA, Hanfling B, Gomez A. Multiple global radiations in tadpole shrimps challenge the concept of 'living fossils. PeerJ. 2013;1:e62.

66. Webster AJ, Payne RJH, Pagel M. Molecular phylogenies link rates of evolution and speciation. Science. 2003;301:478.

67. Gillooly JF, Allen AP, West GB, Brown JH. The rate of DNA evolution: effects of body size and temperature on the molecular clock. Proc Natl Acad Sci U S A. 2005;102:140-5.

68. Welch JJ, Bininda-Emonds ORP, Bromham L. Correlates of substitution rate variation in mammalian protein-coding sequences. BMC Evol Biol. 2008;8:53.

69. Fontanillas $E$, Welch JJ, Thomas JA, Bromham L. The influence of body size and net diversification rate on molecular evolution during the radiation of animal phyla. BMC Evol Biol. 2007:7:95.

70. Thomas JA, Welch JJ, Lanfear R, Bromham L. A generation time effect on the rate of molecular evolution in invertebrates. Mol Biol Evol. 2010;27: $1173-80$.

71. Hebert PDN, Remigio EA, Colbourne JK, Taylor DJ, Wilson CC. Accelerated molecular evolution in halophilic crustaceans. Evolution. 2002;56:909-26.

72. Sueoka N. Directional mutation pressure, mutator mutations, and the dynamics of molecular evolution. J Mol Evol. 1993:37:137-53.

73. Friedrich M, Tautz D. An episodic change of rDNA nucleotide substitution rate has occurred during the emergence of the insect order Diptera. Mol Biol Evol. 1997;14:644-53.

\section{Publisher's Note}

Springer Nature remains neutral with regard to jurisdictional claims in published maps and institutional affiliations.

Ready to submit your research? Choose BMC and benefit from:

- fast, convenient online submission

- thorough peer review by experienced researchers in your field

- rapid publication on acceptance

- support for research data, including large and complex data types

- gold Open Access which fosters wider collaboration and increased citations

- maximum visibility for your research: over $100 \mathrm{M}$ website views per year

At $\mathrm{BMC}$, research is always in progress.

Learn more biomedcentral.com/submissions 